- Over 10 months, 156 referrals, 78 of whom accessed the service

- Average 18 patients on waiting list

- $72 \%$ average attendance/week

- 71\% malignancy; 29\% non-malignant condition

- IPOS data shows mixed scores but tool clinically useful for identifying patients' unmet needs

- Quality of life increased from 3.0 to 4.3 despite overall deteriorating health

- Patients/carers report Day Hospice makes a significant difference with most saying it gives 'a lot of benefit'

- Patterns in phase of illness have emerged and may help to guide decision making regarding follow-up

- $100 \%$ patients discharged from hospice, had consecutive 'stable' status throughout care

- All patients needing specialist follow-up had 'deteriorating' or 'unstable' phase during programme.

Conclusions The redesign has been successful and patient feedback good. Measuring hospice outcomes remains challenging but our experience of the OACC suite has been positive and we are now implementing Karnofsky. A clearer pattern, particularly with IPOS may emerge with more data. However, we may need to wait to benchmark against other similar services or a baseline population.

\section{P-205 THE POSITIVE IMPACT OF A LIVING WELL SERVICE OFFERED WITHIN A PALLIATIVE DAY HOSPICE SETTING}

Sarah Kerry. Ashgate Hospicecare, Chesterfield, UK

\subsection{6/bmispcare-2017-hospice.230}

Background Patients attend Day Hospice for a limited period enabling monitoring and management of symptoms. Once symptoms are managed, the discharge process has proven difficult. The beneficial support and care patients receive mean they are reluctant to terminate attendance, causing anxiety and distress for patients and families.

Aims

- Initiate a structured programme for patients whose symptoms were stable and not requiring specialist medical palliative care

- Help facilitate discharge while empowering patients with information and education

- Utilise the service as a step up or step down tool, for patients that may deteriorate or stabilise, enabling us to offer a flexible, responsive service.

Methods Patients identified by the multi-disciplinary team from the cohort attending Day Hospice that no longer required specialist medical input. Programme is nurse-led with input from the multi-disciplinary team. These patients were reluctant to be discharged as felt they benefitted emotionally and psychologically from the social aspect of the service. A sixteen-week plan was devised which included optional education sessions and circulated to potential attendees. Activities were also available which patients could access if desired. A drop-in session is available on completion of the 16 week programme, ensuring an open door policy to hospice services or enabling people to access social contact. Patients can now join the programme at any point and can be referred directly from other professionals.
Results The pilot is still continuing and evaluation has been ongoing and positive. (Pilot completes 22/6/17). Everyone has benefitted from the education sessions. Patients seem reassured by the offer of drop-in sessions and expressed a desire to attend these.

Conclusion By offering information and tools needed to feel empowered to live well independently with the option of drop- in sessions, appears to be a successful way of supporting patients' discharge from the day hospice.

\section{P-206 PROACTIVE DISCHARGE - A PERSON CENTRED APPROACH TO WELLBEING WITHIN A HOSPICE DAY SERVICE}

Lynsay Bain, Janice Allan. Kilbryde Hospice, East Kilbride, UK

10.1136/bmispcare-2017-hospice.231

Background Feedback demonstrated patients felt unprepared for discharge from a hospice Day-Service. Staff sought a more effective method to support patients to discharge. A Wellbeing Group (WG) was created focusing on enhancing knowledge and self-empowerment using a person-centred approach. This created an inclusive and positive discharge. This approach supports the Scottish Government's vision of Realistic Medicine, putting patients at the centre of decision-making.

Aim The WG aims to empower patients using self-management techniques, enabling patients to make shared decisions. This reduces dependency on hospice and statutory services and focuses on what matters most to the patient.

Methodology A prospective study was planned to identify outcomes of the WG. Sample size $\mathrm{n}=17$, with 16 completing. Sample aged $54-74$, female $80 \%$, male $20 \%$, analysis was carried out by two authors. Outcomes were evaluated through the use of evaluation, Patient Activation Measure - PAM (Hibbard \& Gilburt, 2014) and Views on Care - VoC (Witt et al., 2015).

Results 100\% felt benefit from attending WG.

$92.3 \%$ increased confidence.

$84.6 \%$ improvement in main problems and concerns.

$84.6 \%$ learned techniques that enhanced physical and mental wellbeing.

$73.3 \%$ improvement in the response to the question: 'when all is said and done, I am the person who is responsible for taking care of my health.'

$69.2 \%$ improvement in quality of life.

Patient quote, 'This group has certainly improved my confidence and wellbeing.'

Conclusion Kilbryde Hospice has tailored service delivery according to patient feedback introducing a new and innovative WG. This has shown a positive impact on individuals. All nine PAM questions and evaluation results demonstrated improvement in many areas. Patients felt more prepared for discharge, confident they had the skills to take an active role in their own health and wellbeing. On reflection, PAM could be carried out earlier to ensure WG was the defining factor in improvement. 